ఠ

Open Access Full Text Article

REVIEW

\title{
A clinician's perspective: novel oral anticoagulants to reduce the risk of stroke in nonvalvular atrial fibrillation - full speed ahead or proceed with caution?
}

\author{
This article was published in the following Dove Press journal: \\ Vascular Health and Risk Management \\ 21 August 2014 \\ Number of times this article has been viewed
}

\author{
Eugene Yang \\ Division of Cardiology, University \\ of Washington Medical Center, \\ Seattle, WA, USA
}

\begin{abstract}
Over the past few years, three novel oral anticoagulants, dabigatran, rivaroxaban, and apixaban, have been approved in the USA and Europe to reduce the risk of stroke or systemic embolism in patients with nonvalvular atrial fibrillation, and the results of a Phase III trial for a fourth novel oral anticoagulant, edoxaban, have recently been published. The aim of this review is to examine this indication from a clinician's perspective, highlighting efficacy and safety results from the major trials with these novel oral agents. Clinical issues regarding bleeding, monitoring, and reversal are discussed, along with requirements to consider when interrupting treatment with a novel oral anticoagulant for the purpose of transitioning to another anticoagulant and prior to cardioversion, ablation, percutaneous coronary intervention, or emergency surgery. The cost-effectiveness of each of the approved novel oral anticoagulants is reviewed, and the author provides recommendations for selecting appropriate patients for these agents.
\end{abstract}

Keywords: apixaban, dabigatran, rivaroxaban, edoxaban, anticoagulant reversal, anticoagulant monitoring

\section{Introduction}

Atrial fibrillation (AF) is the most common sustained cardiac arrhythmia, and if left untreated, increases the risk of stroke by five-fold. ${ }^{1-3}$ In 2010, there were an estimated 5.2 million people with AF in the USA, and as the prevalence of AF increases with age, this figure is expected to more than double by 2020, largely as a result of the aging population. . $^{23}$

Oral vitamin K antagonists (VKAs), such as warfarin, have been the mainstay of treatment to reduce the risk of stroke in patients with nonvalvular AF and have been shown to reduce this risk by at least two thirds compared with placebo. ${ }^{4}$ Despite this beneficial effect, VKAs have a narrow therapeutic window and a highly variable dose response attributable to both genetic and clinical factors. ${ }^{5}$ Other elements that influence the anticoagulant effects of VKAs include interactions with prescription and nonprescription drugs and dietary intake of vitamin $\mathrm{K} .{ }^{5}$ Consequently, close monitoring of the international normalized ratio (INR) is essential to ensure that patients are within the appropriate therapeutic range to prevent stroke and to avoid an elevated risk of bleeding. However, frequent and lifelong monitoring, which is a major burden to patients and their families, in addition to concerns about bleeding, particularly intracranial hemorrhage, may have led to significant underutilization of VKAs for stroke prevention, especially in the elderly population.
Correspondence: Eugene Yang Division of Cardiology, University of Washington Medical Center, 1959 NE Pacific Street, Box 356005 Seattle, WA 98195 , USA

$\mathrm{Tel}+\mathrm{I} 2065986819$

Fax +l 2065980445

Email eyang0।@uw.edu 
More than five decades after the approval of warfarin, novel oral anticoagulants (NOACs), ie, dabigatran (a direct thrombin inhibitor) and rivaroxaban, apixaban, and edoxaban (factor Xa inhibitors), have been developed. These NOACs have more predictable pharmacokinetic profiles than warfarin, fewer dietary and drug interactions, and no requirement for routine anticoagulant monitoring (Table 1). ${ }^{6-10}$ Following rigorous Phase III clinical trials (Table 2), ${ }^{11-14}$ dabigatran, rivaroxaban, and apixaban were all approved in Europe and the USA to reduce the risk of stroke or systemic embolism in patients with nonvalvular $\mathrm{AF}^{6-8}$ after all three showed either similar or increased efficacy for reduction of the risk of stroke or systemic embolism compared with warfarin, and a decreased risk of intracranial hemorrhage (Table 3). ${ }^{1-15}$ The results of the Phase III trial for edoxaban have recently been published (Table 3) ${ }^{16}$ however, edoxaban is still under consideration for approval. While apixaban was swiftly approved in Europe, the US Food and Drug Administration (FDA) delayed approval twice, citing the need for additional information on data management and verification in the ARISTOTLE (Apixaban for Reduction In Stroke and Other ThromboemboLic Events in atrial fibrillation) trial, particularly with regard to potential errors in dispensing study medication, which could have affected study outcomes. ${ }^{17}$ Further investigation confirmed the originally reported results, and the FDA recommended approval of apixaban in December 2012. ${ }^{17,18}$

The benefits of the NOACs over warfarin have been partially offset by a lack of reversal agents and an inability to precisely monitor their anticoagulant effects. The purpose of this review is to examine the role of NOACs in reducing the risk of stroke in patients with nonvalvular AF from a clinician's perspective, highlighting results from the major trials and discussing important issues related to their use in clinical practice.

\section{Efficacy and safety of NOACs in Phase III trials Dabigatran}

The efficacy and safety of the direct thrombin inhibitor, dabigatran (150 mg twice daily and $110 \mathrm{mg}$ twice daily) were investigated in the RE-LY (Randomized Evaluation of Long-Term Anticoagulation Therapy) trial (Table 3), the design of which is shown in Table $2 .{ }^{11}$ The approved dosages of dabigatran vary between markets; the FDA-approved dose is $150 \mathrm{mg}$ (75 mg in patients with severe renal impairment), ${ }^{7}$ while both the 150 $\mathrm{mg}$ and $110 \mathrm{mg}$ doses are approved in Europe. ${ }^{19}$ Dabigatran $150 \mathrm{mg}$ twice daily was superior to warfarin $(P<0.001)$ for reduction of the risk of stroke or systemic embolism, with a similar risk of major bleeding between groups $(P=0.31)$. The dabigatran $110 \mathrm{mg}$ twice daily dosage was noninferior to warfarin in reducing the risk of stroke or systemic embolism $(P<0.001)$, with a significantly lower risk of major bleeding $(P=0.003)$. The risk of ischemic stroke was significantly lower with dabigatran $150 \mathrm{mg}$ than with warfarin (relative risk 0.76 , $95 \%$ confidence interval $[\mathrm{CI}] 0.60-0.98 ; P=0.03$ ), but was similar in both groups when dabigatran $110 \mathrm{mg}$ was compared with warfarin (relative risk $1.11,95 \%$ CI $0.89-1.40 ; P=0.35$ ). Compared with warfarin, the risk of intracranial hemorrhage was lower $(P<0.001)$ for both dabigatran doses. The risk of gastrointestinal bleeding was higher with dabigatran $150 \mathrm{mg}$ twice daily than with warfarin $(P<0.001)$, but was similar in the dabigatran $110 \mathrm{mg}$ twice daily and warfarin groups $(P=0.43)$. There was a nonsignificant trend toward reduced risk of mortality with dabigatran $150 \mathrm{mg}$ versus warfarin $(P=0.051)$; however, this trend did not occur with the dabigatran $110 \mathrm{mg}$ twice daily dosage $(P=0.13)$. The only adverse event significantly more common with dabigatran than with warfarin was dyspepsia $(11.8 \%, 11.3 \%$, and $5.8 \%$ for the dabigatran $110 \mathrm{mg}, 150 \mathrm{mg}$, and warfarin groups, respectively). ${ }^{11}$ The risk of myocardial infarction (MI) was higher with dabigatran than with warfarin, but was not statistically significant for either comparison $(P=0.09$ and $P=0.12$, respectively, for the $110 \mathrm{mg}$ and $150 \mathrm{mg}$ twice daily dosages). ${ }^{15}$ A meta-analysis of seven dabigatran trials across indications also found a nonsignificant increase in the risk of MI or acute coronary syndrome (27\% higher in dabigatran-treated patients; $P=0.05) .{ }^{20}$ Another analysis of the RE-LY data found no statistically significant differences in event rates with either dabigatran dosage versus warfarin when using aggregated cardiac events (eg, MI as well as unstable angina, percutaneous coronary intervention, and cardiac arrest). ${ }^{21}$

\section{Rivaroxaban}

The efficacy and safety of the factor Xa inhibitor rivaroxaban were investigated in ROCKET-AF (Rivaroxaban Once Daily Oral Direct Factor Xa Inhibition Compared with Vitamin K Antagonism for Prevention of Stroke and Embolism Trial in Atrial Fibrillation; Table 3). ${ }^{14}$ The trial design is shown in Table 2. Patients who were enrolled in ROCKET-AF were at a higher risk of stroke than those in

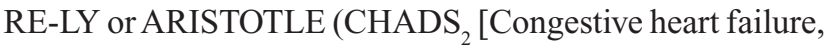
Hypertension, Age $\geq 75$ years, Diabetes mellitus, prior Stroke, transient ischemic attack, or central nervous system thromboembolism \{doubled\}]; score 3.5 versus 2.1 in RE-LY and ARISTOTLE). A once-daily dose of rivaroxaban (20 mg) 


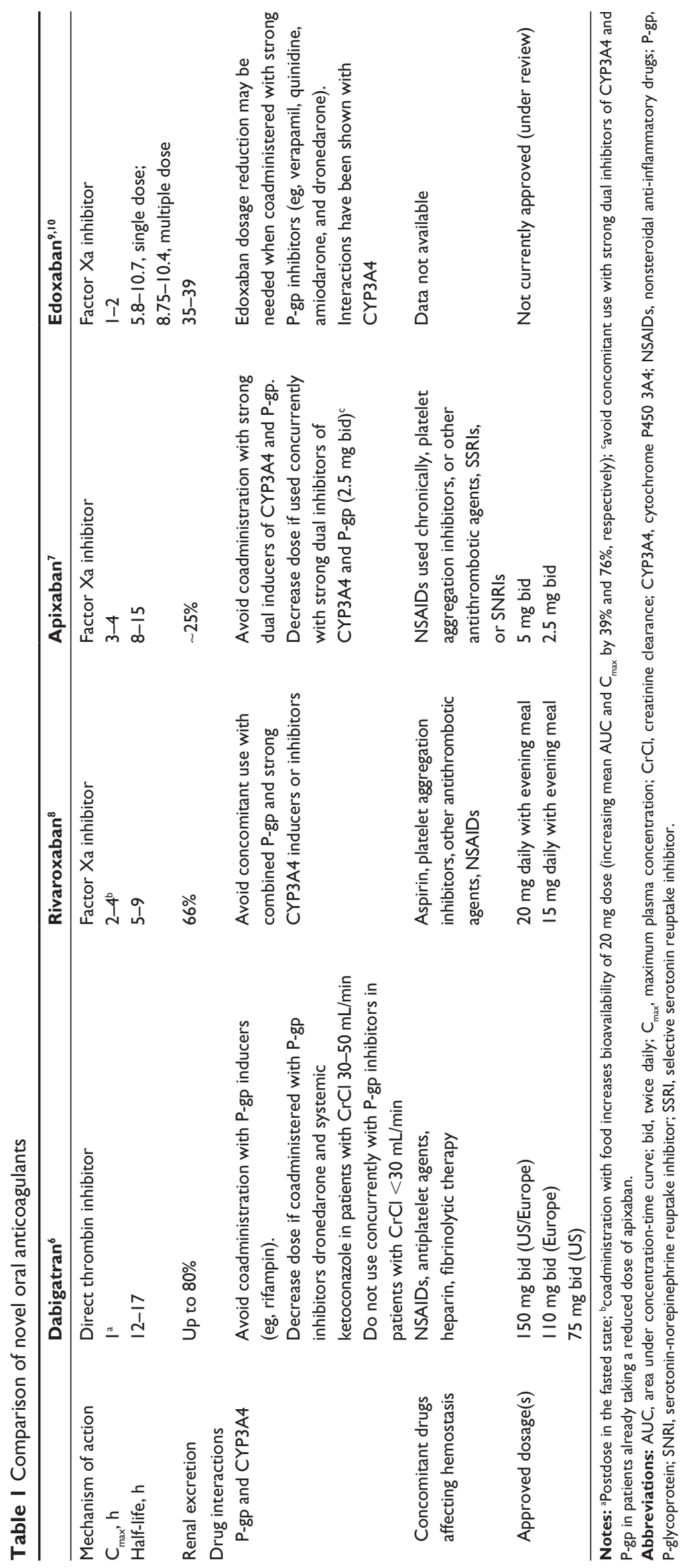


Table 2 Comparison of novel oral anticoagulant trials

\begin{tabular}{|c|c|c|c|c|}
\hline & Dabigatran"I & Rivaroxaban $^{14}$ & Apixaban 12,13 & Edoxaban $^{16}$ \\
\hline Key clinical trial(s) & RE-LY & ROCKET-AF & AVERROES, ARISTOTLE & ENGAGE-AF TIMI-48 \\
\hline Trial design & $\begin{array}{l}\text { Randomized, double-blind, } \\
\text { prospective, open-label } \\
\text { (PROBE) }\end{array}$ & $\begin{array}{l}\text { Randomized, prospective } \\
\text { double-blind, double- } \\
\text { dummy }\end{array}$ & $\begin{array}{l}\text { Randomized, prospective } \\
\text { double-blind, double- } \\
\text { dummy }\end{array}$ & $\begin{array}{l}\text { Randomized, } \\
\text { prospective, double- } \\
\text { blind, double-dummy }\end{array}$ \\
\hline Primary efficacy endpoint & Stroke or SE & Stroke or SE & Stroke or SE & Stroke or SE \\
\hline Primary safety endpoint & Major bleeding (ISTH) & $\begin{array}{l}\text { Major (ISTH)/CRNM } \\
\text { bleeding }\end{array}$ & $\begin{array}{l}\text { Major bleeding } \\
\left(\text { AVERROES, }{ }^{a} \text { ARISTOTLEa) }\right.\end{array}$ & Major bleeding (ISTH) \\
\hline Trial dose & $150 \mathrm{mg}$ bid or $110 \mathrm{mg}$ bid & $20 \mathrm{mg} / \mathrm{d}$ & $5 \mathrm{mg}$ bid & $\begin{array}{l}60 \mathrm{mg} / \mathrm{d} \\
30 \mathrm{mg} / \mathrm{d}\end{array}$ \\
\hline $\begin{array}{l}\text { Reduced dose for } \\
\text { selected patients }\end{array}$ & NA & $\begin{array}{l}15 \mathrm{mg} / \mathrm{d} \\
(\mathrm{CrCl} 30-49 \mathrm{~mL} / \mathrm{min})\end{array}$ & $2.5 \mathrm{mg}$ bid $^{\mathrm{b}}$ & $\begin{array}{l}\text { Dose halved in } \\
\text { selected patients }\end{array}$ \\
\hline Mean $\mathrm{CHADS}_{2}$ score & 2.1 & 3.5 & 2.1 & Not stated \\
\hline Mean TTR, \% & 64 & 55 & 62.2 & 64.9 \\
\hline
\end{tabular}

Notes: a Defined according to ISTH criteria, adjusted for the decrease in hemoglobin level if a blood transfusion is required; ${ }^{b}$ reduced dose if patients met two of the following: age $\geq 80$ years, body weight $\leq 60 \mathrm{~kg}$, or serum creatinine level $\geq 1.5 \mathrm{mg} /$ day; 'for patients in either group the dose was halved if any of the following symptoms were present at the time of randomization or at any point during the study: estimated creatinine clearance $30-50 \mathrm{~mL} / \mathrm{min}$, body weight $\leq 60 \mathrm{~kg}$, or the concomitant use of verapamil or quinidine (potent P-glycoprotein inhibitors). A protocol amendment mandated similar dose modification in the case of concomitant dronedarone use.

Abbreviations: $\mathrm{CHADS}_{2}$, Congestive heart failure, Hypertension, Age $\geq 75$ years, Diabetes, Stroke (doubled); CrCl, creatinine clearance; CRNM, clinically relevant nonmajor; ISTH, International Society on Thrombosis and Haemostasis; NA, not applicable; SE, systemic embolism; TTR, time in therapeutic range; ARISTOTLE, Apixaban for Reduction In Stroke and Other ThromboemboLic Events in atrial fibrillation; AVERROES, Apixaban Versus Acetylsalicylic acid (ASA) to Prevent Strokes; RE-LY, Randomized Evaluation of Long-Term Anticoagulation Therapy; ROCKET-AF, Rivaroxaban versus Warfarin in Nonvalvular Atrial Fibrillation; ENGAGE-AF TIMI-48, Edoxaban versus Warfarin in Patients with Atrial Fibrillation; bid, twice daily.

was used, with a dose reduction $(15 \mathrm{mg})$ in patients with a creatinine clearance of 30-49 mL per minute. Rivaroxaban was noninferior to warfarin $(P<0.001)$ for reduction in the risk of stroke or systemic embolism in the intent-to-treat population; however, superiority was not shown $(P=0.12)$. The risk of major bleeding was similar in rivaroxaban-treated and warfarin-treated patients $(P=0.58)$. The risk of intracranial hemorrhage was significantly lower with rivaroxaban, but the risk of gastrointestinal bleeding was significantly higher ( $P=0.02$ and $P<0.001$, respectively). The risks of mortality and MI were not significantly different between groups ( $P=0.15$ and $P=0.12$, respectively).

\section{Apixaban}

The efficacy and safety of the factor Xa inhibitor apixaban were investigated in the AVERROES (Apixaban Versus Acetylsalicylic acid [ASA] to Prevent Strokes) and ARISTOTLE trials (Table 3). ${ }^{12,13}$ The trial designs are shown in Table 2. In both Phase III trials for apixaban ( $5 \mathrm{mg}$ twice daily), a reduced dose of $2.5 \mathrm{mg}$ (twice daily) was used if patients met two of the following criteria: age $\geq 80$ years, body weight $\leq 60 \mathrm{~kg}$, or serum creatinine level $\geq 1.5 \mathrm{mg} / \mathrm{dL}$. It should be noted that only a limited number of patients received the lower apixaban dose (AVERROES, 6.0\%; ARISTOTLE, 4.7\%). ${ }^{12,13}$ In AVERROES, the risk of stroke or systemic embolism was significantly lower in the apixaban group than in the aspirin group ( $P<0.001$ for superiority); however, the risk of major bleeding was comparable in the two groups $(P=0.57) .{ }^{12}$ A trend toward lower risk of mortality was observed with apixaban $(P=0.07)$, and the risks of intracranial hemorrhage and gastrointestinal bleeding were similar between groups ( $P=0.69$ and $P=0.71$, respectively). In ARISTOTLE, the risk of stroke or systemic embolism was significantly lower with apixaban than with warfarin ( $P<0.01$ for superiority), and this reduction was primarily driven by a reduction in the risk of hemorrhagic stroke (hazard ratio [HR] 0.51, 95\% CI 0.35$0.75 ; P<0.001)$. The risks of major bleeding $(P<0.001)$, intracranial hemorrhage $(P<0.001)$, and mortality $(P=0.047)$ were significantly decreased with apixaban compared with warfarin. ${ }^{13}$ In AVERROES and ARISTOTLE, the risks of gastrointestinal bleeding ( $P=0.71$ and $P=0.37$, respectively) and $\mathrm{MI}(P=0.37$ and $P=0.59$, respectively) were similar with apixaban versus the two comparators. ${ }^{12,13}$

\section{Edoxaban}

The efficacy and safety of the factor Xa inhibitor edoxaban $(60 \mathrm{mg}$ and $30 \mathrm{mg}$ once daily) were investigated in the ENGAGE AFTIMI 48 trial (Edoxaban versus Warfarin in Patients with Atrial Fibrillation); Table 3). ${ }^{16}$ The trial design is shown in Table 2. The edoxaban dose was halved ( $30 \mathrm{mg}$ and $15 \mathrm{mg}$ once daily) if any of the following conditions were present at the time of randomization or at any point during the study: estimated creatinine clearance $30-50 \mathrm{~mL}$ per minute, body weight $\leq 60 \mathrm{~kg}$, or concomitant use of verapamil or quinidine (potent P-glycoprotein inhibitors). A protocol amendment mandated similar dose modification in the event of concomitant dronedarone use. Both doses (60 mg and $30 \mathrm{mg}$ ) were found to be noninferior to warfarin for reduction in the risk of stroke or systemic embolism 
Table 3 Efficacy and safety outcomes in Phase III trials of novel oral anticoagulants ${ }^{\mathrm{a}}$

\begin{tabular}{|c|c|c|c|c|c|}
\hline & Stroke or SE $(\% / y)$ & $\begin{array}{l}\text { Major } \\
\text { bleeding (\%/y) }\end{array}$ & ICH (\%/y) & $\begin{array}{l}\text { GI bleeding } \\
(\% / y)\end{array}$ & Mortality (\%/y) \\
\hline \multicolumn{6}{|l|}{ RE-LY11,15 } \\
\hline Dabigatran $150 \mathrm{mg}$ bid & 1.11 & 3.32 & 0.30 & 1.51 & 3.64 \\
\hline Dabigatran $110 \mathrm{mg}$ bid & 1.54 & 2.87 & 0.23 & 1.12 & 3.75 \\
\hline Warfarin & 1.71 & 3.57 & 0.74 & 1.02 & 4.13 \\
\hline $\begin{array}{l}\text { RR ( } 95 \% \mathrm{Cl}) \text { : dabigatran } \\
\text { I50 mg vs warfarin }\end{array}$ & $\begin{array}{l}0.65(0.52-0.81) \\
P<0.001\end{array}$ & $\begin{array}{l}0.93(0.8 \mathrm{I}-\mathrm{I} .07) \\
P=0.3 \mathrm{I}\end{array}$ & $\begin{array}{l}0.40(0.27-0.60) \\
P<0.00 \text { I }\end{array}$ & $\begin{array}{l}\text { I.50 (I.I9-I.89); } \\
P<0.001\end{array}$ & $\begin{array}{l}0.88(0.77-1.00) ; \\
P=0.05 \mathrm{I}\end{array}$ \\
\hline $\begin{array}{l}\text { RR }(95 \% \mathrm{Cl}) \text { : dabigatran } \\
\text { I } 10 \mathrm{mg} \text { bid vs warfarin }\end{array}$ & $\begin{array}{l}0.90(0.74-1.10) \\
P=0.30\end{array}$ & $\begin{array}{l}0.80(0.70-0.93) \\
P=0.003\end{array}$ & $\begin{array}{l}0.31(0.20-0.47) \\
P<0.001\end{array}$ & $\begin{array}{l}1.10(0.86-1.41) \\
P=0.43\end{array}$ & $\begin{array}{l}0.91(0.80-1.03) ; \\
P=0.13\end{array}$ \\
\hline \multicolumn{6}{|l|}{ ROCKET-AF ${ }^{4}$} \\
\hline Rivaroxaban & 2.1 & 3.6 & 0.5 & 3.2 & 4.5 \\
\hline Warfarin & 2.4 & 3.4 & 0.7 & 2.2 & 4.9 \\
\hline $\mathrm{HR}(95 \% \mathrm{Cl})$ & $\begin{array}{l}0.88(0.75-1.03) ; \\
P<0.00 \text { I (noninferiority); } \\
P=0.12\end{array}$ & $\begin{array}{l}I .04(0.90-1.20) \\
P=0.58\end{array}$ & $\begin{array}{l}0.67(0.47-0.93) \\
P=0.02\end{array}$ & $P<0.00 I^{b}$ & $\begin{array}{l}0.92(0.82-1.03) ; \\
P=0.15\end{array}$ \\
\hline \multicolumn{6}{|l|}{ ARISTOTLE ${ }^{33}$} \\
\hline Apixaban & 1.27 & 2.13 & 0.33 & 0.76 & 3.52 \\
\hline Warfarin & 1.60 & 3.09 & 0.80 & 0.86 & 3.94 \\
\hline $\mathrm{HR}(95 \% \mathrm{Cl})$ & $\begin{array}{l}0.79(0.66-0.95) \\
P<0.0 \text { I }\end{array}$ & $\begin{array}{l}0.69(0.60-0.80) \\
P<0.001\end{array}$ & $\begin{array}{l}0.42(0.30-0.58) \\
P<0.00 \text { I }\end{array}$ & $\begin{array}{l}0.89(0.70-1.15) \\
P=0.37\end{array}$ & $\begin{array}{l}0.89(0.80-0.99) ; \\
P=0.047\end{array}$ \\
\hline \multicolumn{6}{|l|}{ AVERROES ${ }^{12}$} \\
\hline Apixaban & 1.6 & 1.4 & 0.4 & 0.4 & 3.5 \\
\hline Aspirin & 3.7 & 1.2 & 0.4 & 0.4 & 4.4 \\
\hline $\mathrm{HR}(95 \% \mathrm{Cl})$ & $\begin{array}{l}0.45(0.32-0.62) \\
P<0.00 \text { I }\end{array}$ & $\begin{array}{l}I .13(0.74-1.75) \\
P=0.57\end{array}$ & $\begin{array}{l}0.85(0.38-1.90) \\
P=0.69\end{array}$ & $\begin{array}{l}0.86(0.40-1.86) \\
P=0.71\end{array}$ & $\begin{array}{l}0.79(0.62-1.02) ; \\
P=0.07\end{array}$ \\
\hline \multicolumn{6}{|l|}{ ENGAGE AF TIMI-48 ${ }^{16}$} \\
\hline Edoxaban $60 \mathrm{mg}$ & 1.57 & 2.75 & 0.39 & 1.51 & 3.99 \\
\hline Edoxaban $30 \mathrm{mg}$ & 2.04 & 1.61 & 0.26 & 0.82 & 3.80 \\
\hline Warfarin & 1.80 & 3.43 & 0.85 & 1.23 & 4.35 \\
\hline $\begin{array}{l}\mathrm{HR}(95 \% \mathrm{Cl}) \text { : high dose vs } \\
\text { warfarin }\end{array}$ & $\begin{array}{l}\text { mITT:c } 0.79(0.63-0.99) ; \\
P<0.00 \text { I (noninferiority) } \\
\text { ITT:d } 0.87(0.73-1.04) ; \\
P=0.08\end{array}$ & $\begin{array}{l}0.80(0.7 \mid-0.91) \\
P<0.001\end{array}$ & $\begin{array}{l}0.47(0.34-0.63) \\
P<0.001\end{array}$ & $\begin{array}{l}1.23(1.02-1.50) \\
P=0.03\end{array}$ & $\begin{array}{l}0.92(0.83-1.01) \\
P=0.08\end{array}$ \\
\hline $\begin{array}{l}\mathrm{HR}(95 \% \mathrm{Cl}) \text { : low dose vs } \\
\text { warfarin }\end{array}$ & $\begin{array}{l}\text { mITT:c I.07 (0.87-I.3I); } \\
P=0.005 \text { (noninferiority) } \\
\text { ITT: }:^{\text {I } I . I 3}(0.96-0.34) ; \\
P=0.10\end{array}$ & $\begin{array}{l}0.47(0.4 \mathrm{I}-0.55) \\
P<0.00 \mathrm{I}\end{array}$ & $\begin{array}{l}0.30(0.2 \mathrm{I}-0.43) \\
P<0.00 \mathrm{I}\end{array}$ & $\begin{array}{l}0.67(0.53-0.83) \\
P<0.001\end{array}$ & $\begin{array}{l}0.87(0.79-0.96) \\
P=0.006\end{array}$ \\
\hline
\end{tabular}

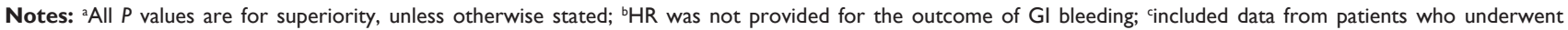
randomization and received at least one dose of study drug during the treatment period (from administration of first dose to either 3 days after receipt of last dose or end

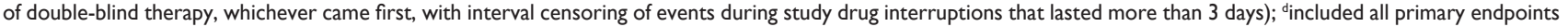
during the overall study period from randomization until the end of double-blind treatment.

Abbreviations: $\mathrm{Cl}$, confidence interval; $\mathrm{Gl}$, gastrointestinal; HR, hazard ratio; ICH, intracranial hemorrhage; ITT, intent-to-treat; mITT, modified intent-to-treat; RR, relative risk; SE, systemic embolism; vs, versus; ARISTOTLE, Apixaban for Reduction In Stroke and Other ThromboemboLic Events in atrial fibrillation; AVERROES, Apixaban Versus Acetylsalicylic acid (ASA) to Prevent Strokes; RE-LY, Randomized Evaluation of Long-Term Anticoagulation Therapy; ROCKET-AF, Rivaroxaban versus Warfarin in Nonvalvular Atrial Fibrillation; ENGAGE-AF TIMI-48, Edoxaban versus Warfarin in Patients with Atrial Fibrillation; bid, twice daily.

(modified intent-to-treat population, $P<0.001$ and $P=0.005$ for noninferiority, respectively; intent-to-treat population, $P=0.08$ and $P=0.10$ for superiority, respectively) and were associated with significantly lower risks of major bleeding $(P<0.001$ and $P<0.001) .{ }^{16}$ The risk of all-cause mortality was significantly reduced with edoxaban $30 \mathrm{mg}$ versus warfarin $(P=0.006)$ but was similar in the edoxaban $60 \mathrm{mg}$ and warfarin groups $(P=0.08) .{ }^{16}$ The risk of major gastrointestinal bleeding was significantly higher with edoxaban $60 \mathrm{mg}$ than with warfarin $(P=0.03)$, but was lower with edoxaban $30 \mathrm{mg}(P<0.001$ versus warfarin). Edoxaban is not discussed further in this review, because neither dose has yet been approved by the FDA or European agencies.

\section{Clinical issues}

Although the RE-LY, ROCKET-AF, and ARISTOTLE studies investigated similar outcomes, only head-to-head comparisons can determine the relative safety of each of these NOACs versus the others. Whereas all treatment arms within a trial are equally affected, one should consider differences in trial design (Table 2), 
such as baseline $\mathrm{CHADS}_{2}$ scores and study drug blinding, before making indirect comparisons between NOACs. Nevertheless, understanding clinical issues, such as bleeding, monitoring, and reversal, may help clinicians select the appropriate treatment to reduce the risk of stroke or systemic embolism in patients with nonvalvular AF. A practical guide for how to manage NOACs in specific clinical situations has been developed by the European Heart Rhythm Association (EHRA). ${ }^{22}$

\section{Bleeding}

Real-world experience and postmarketing data are providing additional insights into the use of NOACs in patients with nonvalvular AF. Since its approval in late 2010, reports have associated dabigatran with serious adverse events, particularly bleeding, most commonly in patients with low body weight, advanced age, or impaired renal function. ${ }^{23-25}$ The information from these reports is consistent with data from a RE-LY subanalysis that identified older age ( $\geq 75$ years) and poor renal function as key predictors of bleeding events with dabigatran treatment. ${ }^{26}$ Subsequent investigations by the FDA and the European Medicines Agency's Committee for Medicinal Products for Human Use concluded that the bleeding risks were consistent with the bleeding rates reported in RE-LY, ${ }^{27,28}$ and the dabigatran prescribing information recommends assessing patient renal function before beginning treatment and as clinically indicated thereafter. ${ }^{6}$ Furthermore, the FDA concluded in a Mini-Sentinel pilot analysis that observed bleeding rates associated with new use of dabigatran were lower than bleeding rates associated with new use of warfarin. ${ }^{28} \mathrm{~A}$ larger study was conducted by the FDA to assess the efficacy and safety of dabigatran versus warfarin in 134,000 Medicare patients, aged 65 years or older, who had received a diagnosis of nonvalvular AF within the 6 months prior to the first dispensing of medication. ${ }^{29}$ The results showed that dabigatran (combined data for $150 \mathrm{mg}$ and $75 \mathrm{mg}$ dosages) was associated with a lower risk of ischemic stroke (adjusted HR 0.80, 95\% CI 0.67-0.96), intracranial hemorrhage (HR $0.34,95 \%$ CI $0.26-0.46$ ), and death (HR 0.86, 95\% CI 0.77-0.96) compared with warfarin. An increased risk of major gastrointestinal bleeding (HR 1.28, 95\% CI 1.14-1.44) was observed; however, the risk of MI was similar between the treatment groups. ${ }^{29} \mathrm{~A}$ real-world, nationwide cohort study was conducted in Denmark that assessed the use of dabigatran (post-approval) in anticoagulant-naive patients with $\mathrm{AF}^{30} \mathrm{In}$ the 14,267 patients identified through registry data, the mean age was 70.8 years, $56.5 \%$ were male, the mean $\mathrm{CHADS}_{2}$ score was 1.16 , and the median follow-up was 10.5 months. ${ }^{30}$ The investigators found that there were similar risks of stroke or systemic embolism and major bleeding with both doses (150 mg and $110 \mathrm{mg}$ ) of dabigatran and with warfarin, and that the risks of mortality, intracranial hemorrhage, pulmonary embolism, and MI were all lower in dabigatran-treated patients. ${ }^{30}$ An additional real-world study from a single hospital in Hong Kong was conducted to compare dabigatran with warfarin (matched by age, sex, and treatment duration) in patients with $\mathrm{AF}^{31}{ }^{31}$ The mean age of the 244 patients recruited was 70.1 years, $54.1 \%$ were male, their mean $\mathrm{CHADS}_{2}$ score was 2.4, and the median treatment duration was 310 days. ${ }^{31}$ The investigators found that the risks of stroke and bleeding (any degree) were similar in dabigatran-treated and warfarintreated patients. ${ }^{31}$ The investigators also found that there was no significant difference in treatment compliance rates between the two groups. ${ }^{31}$

Real-world data on bleeding with rivaroxaban and apixaban are limited, as these therapies have been available for less time than dabigatran, but trial subanalyses are available. In ROCKET-AF, predictors of major bleeding with rivaroxaban included older age, male sex, increased body mass index, diabetes, chronic obstructive lung disease, and worsening renal function. ${ }^{32}$ Risks of major adverse outcomes, including death following a major bleeding event, were similar in patients treated with rivaroxaban and warfarin in ROCKET-AF. ${ }^{33}$ Additionally, rivaroxaban was associated with a lower risk of intracranial hemorrhage. ${ }^{33}$ A subgroup analysis of ARISTOTLE found a lower risk of bleeding in all age categories for apixaban-treated versus warfarin-treated patients, including those aged $\geq 75$ years. ${ }^{13}$ In addition to observing a reduced risk of stroke or systemic embolism regardless of renal function, another analysis from ARISTOTLE found a greater reduction in the relative risk of major bleeding in the apixaban arm versus warfarin arm with worsening renal impairment (creatinine clearance $<50 \mathrm{~mL}$ per minute). ${ }^{34}$ When considering these data, it is important to note that patients with a creatinine clearance $<30 \mathrm{~mL}$ per minute (RE-LY and ROCKET-AF) or $<25 \mathrm{~mL}$ per minute (ARISTOTLE) were excluded from the trials; NOACs should not be used in patients with creatinine clearance $<15 \mathrm{~mL}$ per minute, as there are limited clinical outcomes data to inform on the use of NOACs in such patients. ${ }^{6-8} \mathrm{~A}$ separate analysis of the ARISTOTLE trial found that, compared with warfarin, apixaban was associated with a $31 \%$ reduction in risk of a first major bleeding event, and was associated with fewer intracranial hemorrhages. ${ }^{35}$ Additionally, apixaban was associated with fewer adverse consequences following extracranial hemorrhages, fewer trauma-associated hemorrhages, and a 50\% reduction in fatal events at 30 days in the case of a major hemorrhage. ${ }^{35}$ The 
EHRA guide states that current recommendations on bleeding management are not so much based on clinical experience as expert opinion on laboratory endpoints. ${ }^{22}$ For bleeding that is not life-threatening, the guide suggests that time is the most important reversal strategy for the anticoagulant effects of NOACs because of their short half-lives (Table 1); however, standard supportive measures should also be used, including mechanical compression, surgical hemostasis, fluid replacement, and other hemodynamic support. Data to support how to treat life-threatening bleeding are limited, ${ }^{22}$ and are discussed in more detail in the next two sections.

\section{Monitoring anticoagulant effects}

Due to its narrow therapeutic window, patients taking warfarin require regular monitoring to maintain therapeutic levels (INR 2.0-3.0). ${ }^{5}$ Equivalent monitoring is not required with NOAC treatment and INR is not an effective option for monitoring anticoagulation in NOAC-treated patients. Patients most likely to require anticoagulation for nonvalvular AF are older and have more comorbidities, and therefore may interact with a variety of health care providers. Many of these providers may be unfamiliar with NOACs and assume that INR testing can be done for these agents.
A number of treatment-specific methods for monitoring NOAC treatment are under investigation (Table 4), but they are generally not ready for clinical use and data are limited to a few small studies. ${ }^{36-42}$ These tests differ from those used for warfarin because of their target-specific mechanisms of action (Figure 1). Additionally, for NOACs, the direct anticoagulant activity, rapid onset/offset of anticoagulant effects, and relatively short half-lives make it critically important to know the timing of the last drug dose when evaluating test results.

\section{Dabigatran}

A close linear relationship has been shown between prolongation of ecarin clotting time and plasma concentrations of dabigatran..$^{38,43}$ Ecarin clotting time is the most sensitive assay for determining the anticoagulant effect of dabigatran; however, it is not widely available. Prothrombin time is widely available, but unsuitable as a primary measure of anticoagulant activity. ${ }^{41}$ Measurement of activated partial thromboplastin time (aPTT) at trough levels of dabigatran may be useful for identifying an excess anticoagulant effect but not for precise quantification. ${ }^{6,41}$ The thrombin time assay is useful in detecting very low dabigatran plasma levels, and the diluted thrombin time assay can identify therapeutic and supratherapeutic levels. ${ }^{40,43}$ The dabigatran prescribing

Table 4 Anticoagulant monitoring assays

\begin{tabular}{|c|c|c|c|c|}
\hline & Description & Dabigatran $38,40,41$ & Rivaroxaban $^{36,37,39}$ & Apixaban ${ }^{7,36,42,44}$ \\
\hline Anti-factor $\mathrm{Xa}$ & $\begin{array}{l}\text { Measures factor } X \text { activation directly } \\
\text { using a chromogenic substrate }\end{array}$ & NA & Quantitative & Quantitative \\
\hline $\begin{array}{l}\text { Activated partial } \\
\text { thromboplastin } \\
\text { time (aPTT) }\end{array}$ & $\begin{array}{l}\text { Test for the intrinsic system; measures } \\
\text { kininogen, prekallikrein, XII, XI, IX, } \\
\text { VIII, X, V, and thrombin }\end{array}$ & Qualitative & Not useful & Not useful \\
\hline $\begin{array}{l}\text { Prothrombin time } \\
\text { (PT) }\end{array}$ & $\begin{array}{l}\text { Test of the extrinsic pathway, provides } \\
\text { an overview of VII, X, V, thrombin, } \\
\text { and fibrinogen }\end{array}$ & Not useful & Qualitative & Not useful \\
\hline $\begin{array}{l}\text { Thrombin time } \\
\text { (TT) }\end{array}$ & $\begin{array}{l}\text { Functional test of fibrinogen } \\
\text { concentration and fibrin formation }\end{array}$ & $\begin{array}{l}\text { Qualitative assessment, but } \\
\text { values may be abnormally high } \\
\text { even at clinically insignificant } \\
\text { levels }\end{array}$ & Not useful & Not useful \\
\hline Diluted TTa & $\begin{array}{l}\text { Uses the Hemoclot Thrombin } \\
\text { Inhibitor assay (Hyphen BioMed, } \\
\text { Neuville-sur-Oise, France) }{ }^{40}\end{array}$ & Quantitative analysis & Not useful & Not useful \\
\hline Modified PTa & $\begin{array}{l}\text { Modification of PT assay by adding } \\
\text { calcium chloride to the thromboplastin } \\
\text { reagent to increase assay dynamic } \\
\text { range and improve sensitivity }{ }^{42}\end{array}$ & NA & NA & $\begin{array}{l}\text { More sensitive than } \\
\text { PT and } \text { aPTT }^{41}\end{array}$ \\
\hline Ecarin clotting time ${ }^{a}$ & $\begin{array}{l}\text { Specific assay for thrombin } \\
\text { generation }\end{array}$ & Quantitative & NA & NA \\
\hline $\begin{array}{l}\text { Heptest }^{\circledR a} \\
\text { (American Diagnostica, } \\
\text { Stamford, CT, USA) }\end{array}$ & Quantitative heparin clotting test & NA & Prolonged & $\begin{array}{l}\text { More sensitive than } \\
\text { PT and } \mathrm{APTT}^{42}\end{array}$ \\
\hline
\end{tabular}

Note: ${ }^{a}$ Not widely available.

Abbreviations: NA, data not available; aPTT, activated partial thromboplastin time; PT, prothrombin time; TT, thrombin time. 


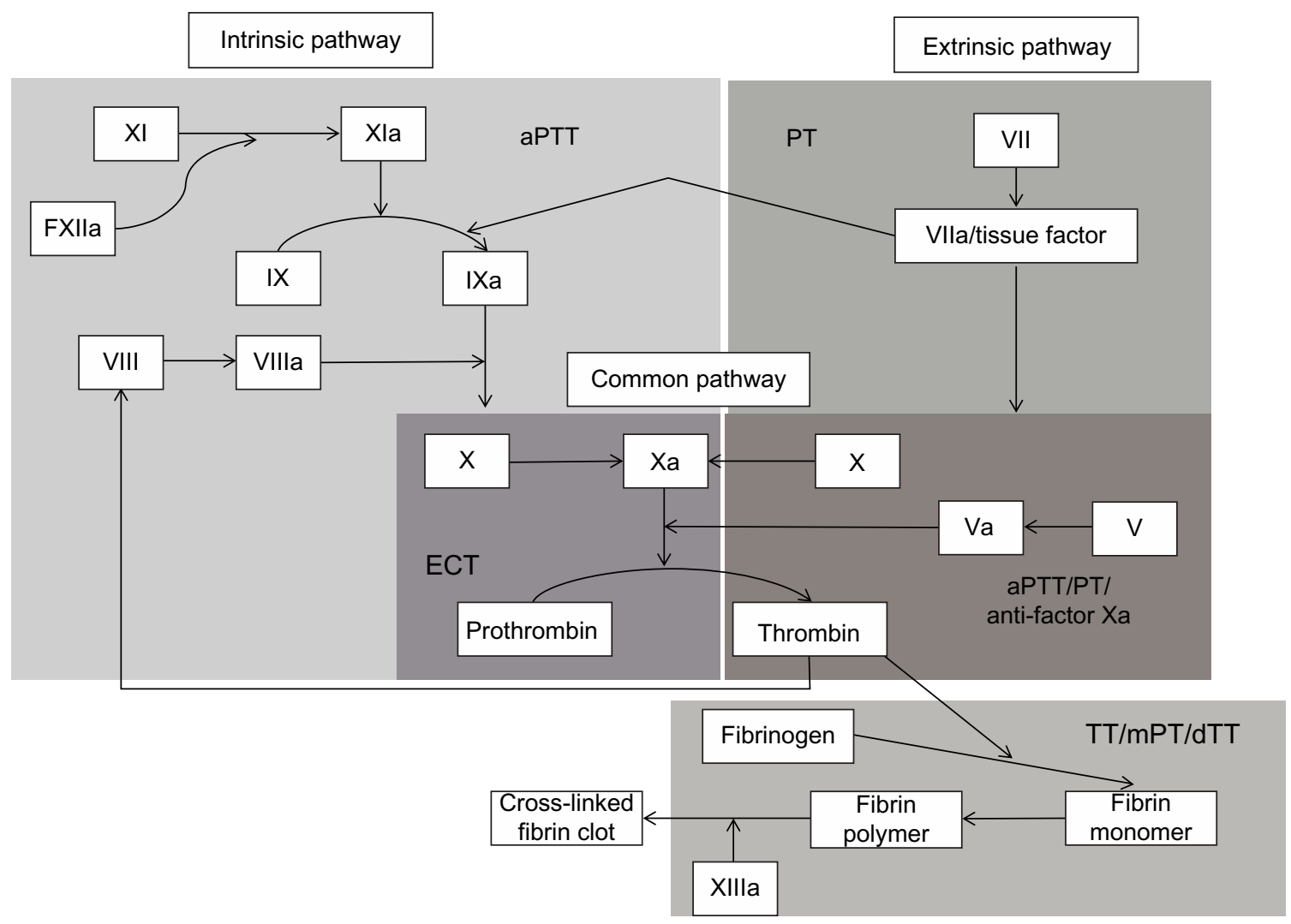

Figure I Sites of action for monitoring assays in the coagulation pathway.

Abbreviations: aPTT, activated partial thromboplastin time; dTT, diluted thrombin time; ECT, ecarin clotting time; mPT, modified prothrombin time; PT, prothrombin time; TT, thrombin time.

information suggests use of aPTT or ecarin clotting time, but not INR, to assess the anticoagulant activity of dabigatran. ${ }^{6}$

\section{Rivaroxaban}

The prothrombin time, aPTT, Heptest ${ }^{\mathbb{}}$ (American Diagnostica, Stamford, CT, USA), and anti-factor Xa assays are all prolonged by rivaroxaban. ${ }^{8}$ Rivaroxaban and other factor Xa inhibitors affect INR measurements, and if treatment with rivaroxaban and warfarin overlap, prothrombin time is not useful for assessing the extent of anticoagulation. The Heptest, which measures inhibition of exogenous factor Xa, may then be a good alternative. The prothrombinase-induced clotting time can also be used, but is not commercially available and offers limited results. The anti-factor Xa assay is the most sensitive and reliable method for assessing the anticoagulant effect of rivaroxaban, and kits with rivaroxaban calibrators and controls are now commercially available. ${ }^{44,45}$

\section{Apixaban}

In human plasma, the anticoagulant effect of apixaban on prothrombin time varies significantly depending on the thromboplastin reagent used, and therefore prothrombin time is not useful for monitoring. ${ }^{7}$ It may, however, have clinical utility in an emergency situation to provide a qualitative assessment of whether there is any anticoagulant effect remaining. Like other factor Xa inhibitors, apixaban affects INR results, thus these measurements may not be useful for determining the extent of anticoagulation when warfarin is coadministered with apixaban. ${ }^{7}$ Although not widely available, the modified prothrombin time and Heptest are more sensitive than prothrombin time and aPTT. ${ }^{42}$ The widely available Rotachrom ${ }^{\circledR}$ heparin chromogenic anti-factor Xa assay (Diagnostica Stago Inc., Parsippany, NJ, USA) appears to be the most sensitive method for measuring apixaban concentrations. ${ }^{44,46}$

\section{Reversal of anticoagulation}

Reversal agents such as protamine, fresh frozen plasma, and 3-factor and 4-factor prothrombin complex concentrate have successfully reversed the anticoagulant effects of heparin derivatives and warfarin. ${ }^{5,47}$ There is no drug-specific antidote to rapidly reverse the drug effect of NOACs or induce hemostasis. Therefore, reduction of drug exposure mostly relies on the short half-lives of these agents (5-17 hours, 
Table 1), which culminate in rapid reductions of their anticoagulant effects compared with warfarin. ${ }^{6-8}$

Dabigatran is dialyzable due to its relatively low ( $\sim 60 \%$ of drug over $2-3$ hours) plasma protein binding, ${ }^{6}$ although data for this treatment approach are limited. The majority of rivaroxaban and apixaban $(92 \%-95 \%$ and $87 \%$, respectively) is protein-bound in plasma, so dialysis is not a viable option to reverse anticoagulation. ${ }^{7,8}$

Fresh frozen plasma may reverse the anticoagulant effects of dabigatran, rivaroxaban, and apixaban, but has shown limited effectiveness in reducing dabigatran-associated intracerebral hematoma in a murine intracranial hemorrhage model. ${ }^{48}$ Activated charcoal has reduced absorption when administered within 8 hours of rivaroxaban or within 6 hours of apixaban ingestion. ${ }^{49,50}$ Prothrombin complex concentrates have been suggested as possible therapies to reverse NOAC anticoagulation. In healthy volunteers, nonactivated prothrombin complex concentrates normalized coagulation test results in patients taking rivaroxaban but not in patients taking dabigatran. ${ }^{51}$ However, activated prothrombin complex concentrate reduced bleeding times in dabigatran-treated rats. ${ }^{41}$

A study of healthy volunteers $(n=10)$ exposed to rivaroxaban and dabigatran found equivocal results for prothrombin complex concentrates, recombinant factor VIIa (rFVIIa), and FEIBA $^{\circledR}$ (anti-inhibitor coagulant complex, Baxter Healthcare Corporation, Deerfield, IL, USA). ${ }^{52}$ A recent in vitro investigation found variable effectiveness of prothrombin complex concentrate, activated prothrombin complex concentrate, and rFVIIa in reversing alterations of hemostatic mechanisms induced by apixaban. ${ }^{53}$ Efficacy was greatest for rFVIIa and prothrombin complex concentrate, depending on the outcome measured. A Phase II proof-of-concept study has shown that the novel recombinant protein PRT064445 produces rapid, sustained, dose-related reversal of the anticoagulant effects of both apixaban and rivaroxaban..$^{54,55}$

\section{Permanent discontinuation of NOACs}

Some patients will require permanent discontinuation of a NOAC and will transition back to warfarin, and as with transitions from warfarin to a NOAC, adequate anticoagulation must be maintained. The FDA has issued boxed warnings for the three approved NOACs regarding the increased risk of thrombotic events following discontinuation of treatment, and recommends considering another anticoagulant when cessation of treatment is unrelated to pathological bleeding. ${ }^{6-8}$ This warning ensued from the observation of increased frequency of thrombotic events following discontinuation of rivaroxaban and apixaban at the end of the ROCKET-AF and ARISTOTLE trials, respectively; however, additional analyses are needed to improve current understanding of these findings. ${ }^{14,56-59}$ No clinical trial data are available on the conversion of patients from NOACs to warfarin, and it is not yet known which patients are at highest risk for thrombotic events following discontinuation of treatment. A suggested approach is to discontinue NOAC treatment and begin a parenteral anticoagulant and warfarin at the time the next dose would have been taken, discontinuing the parenteral anticoagulant when the INR is within the therapeutic range. ${ }^{6-8}$ The American College of Chest Physicians guidelines define the risk of thromboembolism as high for patients with a $\mathrm{CHADS}_{2}$ score of 5 or 6 , a recent ( $<3$ months) stroke or transient ischemic attack, or rheumatic valvular heart disease; moderate for patients with a $\mathrm{CHADS}_{2}$ score of 3 or 4 ; and low for patients with a $\mathrm{CHADS}_{2}$ score of 0 to 2 and no prior stroke or transient ischemic attack. Although no specific data are available for NOACs, this risk stratification scheme could provide guidance when considering which patients should receive bridging therapy. ${ }^{60}$ Additional guidance on switching between specific antithrombotic regimens is provided in the EHRA practical guide. ${ }^{22}$ Careful patient monitoring is recommended when switching between anticoagulants, as a recent real-world study showed that patients who switched to dabigatran had an increased risk of MI when compared with those who remained on warfarin therapy during the early period after transition. ${ }^{61}$

\section{Transition between NOACs}

An area of uncertainty in clinical care is the lack of practical guidance on how to transition patients from one NOAC to another. This is driven by the absence of head-to-head trials between NOACs. If patients develop side effects from a NOAC and wish to convert to another one, no published protocols are available to assist practitioners with this process. Since the half-lives of the NOACs are between 5 and 17 hours (Table 1), it is reasonable to discontinue NOACs dosed twice daily, such as dabigatran or apixaban, after taking the evening dose and starting the first dose of the new agent the following morning. If taking rivaroxaban once daily, it is sensible to advise patients to take their daily evening dose and start one of the NOACs dosed twice daily the next evening. As real-world experience accumulates with NOACs and conversion between agents becomes more common, guidelines will likely emerge to set standards for this transition. 


\section{Temporary interruption of NOACs}

Clinical situations such as catheter ablation and emergency surgery may require temporary interruption of anticoagulant treatment. Providers familiar with managing patients on warfarin may have uncertainty when faced with the same situations in patients treated with a NOAC, where limited data are available to guide treatment decisions. The American College of Cardiology guidelines state that in patients with $\mathrm{AF}$ (without a mechanical heart valve) who require interruption of warfarin or NOAC treatment, the need for bridging therapy with unfractionated or low-molecular-weight heparin should balance the risks of stroke and bleeding and the duration of time a patient will not be anticoagulated. ${ }^{62}$ The prescribing information for apixaban states that bridging anticoagulation during the 24-48 hours after stopping apixaban and prior to elective surgery or invasive procedures is not generally required and that apixaban should be restarted as soon as adequate hemostasis has been established. ${ }^{7}$ No guidance on whether or not to use bridging therapy has been provided for dabigatran or rivaroxaban.

\section{Catheter ablation}

Patients with AF undergoing catheter ablation are generally anticoagulated prior to the procedure. A recent trend has been to continue warfarin uninterrupted through the ablation periprocedural period, but appropriate timing for NOAC discontinuation remains uncertain. Data for NOAC discontinuation prior to ablation are limited. Studies investigating use of NOACs in patients undergoing catheter ablation have been conducted using differing trial designs, which makes it difficult to interpret their conflicting results..$^{57,63-67}$ In a multicenter registry of patients $(\mathrm{n}=290)$ undergoing radiofrequency ablation for nonvalvular AF, warfarin therapy was not interrupted, whereas dabigatran was held on the morning of the procedure and resumed within 3 hours of hemostasis. ${ }^{57}$ Rates of total and major bleeding were significantly higher in the dabigatran group than in the warfarin group ( $14 \%$ versus $6 \%, P=0.031$, and $6 \%$ versus $1 \%, P=0.019$, respectively). Conversely, a single-center study $(n=123)$ in which warfarin was discontinued 5 days before ablation and dabigatran was stopped 36-60 hours before the procedure based on glomerular filtration rate recorded no hemorrhagic or thrombotic events in dabigatran-treated patients ${ }^{67} \mathrm{~A}$ prospective observational study of 259 real-world patients with nonvalvular AF undergoing catheter ablation was performed to assess the longer-term safety, efficacy, and acceptance of NOACs. ${ }^{63}$ Patients were treated for at least 3 months with NOACs post-ablation; $38 \%$ received dabigatran $110 \mathrm{mg}$ twice daily, $56 \%$ received dabigatran $150 \mathrm{mg}$ twice daily, and $6 \%$ received rivaroxaban $20 \mathrm{mg}$ once daily. Of the four periprocedural thromboembolic and major bleeding complications $(1.5 \%)$, none occurred in patients treated with NOACs prior to ablation, and no stroke, systemic embolism, or major bleeding events occurred during long-term follow-up. Hence, the authors concluded that, in this population, anticoagulation with NOACs following AF catheter ablation was safe and effective at long-term follow-up. ${ }^{63}$

In addition to these studies, a meta-analysis was conducted to evaluate the efficacy and safety of dabigatran in 5513 patients (dabigatran, $n=2137$; warfarin, $n=3376$ ) undergoing catheter ablation. ${ }^{65}$ The results showed that the risk of stroke or transient ischemic attack (odds ratio 3.94; 95\% CI 1.54-10.08) and of all thromboembolic events was higher with dabigatran than with warfarin (odds ratio 2.81; 95\% CI 1.23-6.45), with no major differences in risk of major bleeding (odds ratio $0.99 ; 95 \%$ CI $0.55-1.78$ ) ${ }^{65}$

A study evaluating the risk of bleeding and thromboembolic complications associated with NOAC use during ablation compared the use of uninterrupted warfarin $(n=114)$, dabigatran $(\mathrm{n}=89)$, and rivaroxaban $(\mathrm{n}=98) \cdot{ }^{66}$ Dabigatran was held for 24 hours and rivaroxaban for 36 hours prior to the procedure; heparin infusion was initiated 6 hours post-procedure, and NOAC treatment was resumed the morning after the procedure. Two thromboembolic events and 17 bleeding events occurred; however, there was no significant difference between the groups with regard to the combined thromboembolic/bleeding risk (warfarin, 6.2\%; dabigatran, 6.7\%; rivaroxaban, $6.0 \% ; P=0.82$ ). A further single-center study compared VKAs $(\mathrm{n}=192)$, rivaroxaban $(\mathrm{n}=188)$, and dabigatran $(\mathrm{n}=176)$ in patients undergoing catheter ablation. ${ }^{64}$ In the 30 days following ablation, the rates of thromboembolic events (VKA, 2.1\%; dabigatran, $0.6 \%$; rivaroxaban, $1.1 \% ; P=0.410)$ and major bleeding (VKA, $4.2 \%$; dabigatran, $1.1 \%$; rivaroxaban, $1.6 \% ; P=0.112$ ) were low and comparable between treatments. ${ }^{64}$

Based on the available data, the EHRA guide suggests that to reduce bleeding and catheter ablation complications in patients taking NOACs, a strategy of bridging and restarting anticoagulation may be safe when appropriately executed. They caution, however, that an aggressively shortened periprocedural cessation of NOACs and/or no bridging may be less safe when compared with ablation performed with uninterrupted VKA administration and a therapeutic INR between 2.0 and 3.0. ${ }^{22}$

\section{Surgical procedures}

Approximately $10 \%$ of patients receiving oral anticoagulants require interruption of treatment for surgery or elective 
invasive procedures. ${ }^{60}$ Standard guidance is to withhold anticoagulant treatment for at least 4-6 half-lives prior to surgery. In addition, the EHRA guide suggests considering the bleeding risk of surgery when deciding how to manage anticoagulation treatment. ${ }^{22}$ Dabigatran should be discontinued 1-2 days prior to invasive or surgical procedures in patients with creatinine clearance $\geq 50 \mathrm{~mL}$ per minute, or $3-5$ days prior in patients with creatinine clearance $<50 \mathrm{~mL}$ per minute. ${ }^{6}$ Rivaroxaban should be discontinued at least 24 hours prior to surgical or other procedures. ${ }^{8}$ Apixaban should be discontinued at least 48 hours prior to elective surgery or invasive procedures with a moderate-to-high risk of unacceptable or clinically relevant bleeding, and at least 24 hours before similar procedures with a low risk of bleeding or where the bleeding would be noncritical in location and easily controlled. ${ }^{7}$

In a subanalysis of RE-LY, the risks of periprocedural complications, perioperative bleeding, and thrombotic events in patients taking dabigatran were comparable with those in patients taking warfarin, even among patients having major or urgent surgery. Furthermore, dabigatran facilitated a shorter interruption of anticoagulation than warfarin. ${ }^{68}$ In ROCKET-AF, temporary discontinuation of rivaroxaban was equivalent to warfarin in terms of stroke risk, but this analysis did not evaluate differences in perioperative bleeding or thrombotic risks related to invasive procedures (eg, major surgery). ${ }^{58}$ In ARISTOTLE, rates of stroke and major bleeding during the 30 days following procedures were low and comparable in warfarin-treated and apixabantreated patients. ${ }^{69}$

\section{Other clinical scenarios Cardioversion}

The European Society of Cardiology guidelines suggest that patients with an AF duration $>48$ hours who undergo elective cardioversion should receive anticoagulation for at least 3 weeks prior to the procedure, or transesophageal echocardiography should be performed to rule out left atrial thrombus if anticoagulant treatment is less than the mandatory 3 weeks. ${ }^{1}$ After cardioversion, anticoagulation should be continued for at least 4 weeks. Cardioversion protocols for patients taking NOACs are lacking. Currently, information is limited to the protocols and results from the major NOAC trials. Patients in RE-LY continued the study drug prior to cardioversion, unless the investigator deemed another approach was necessary. To exclude the presence of left atrial thrombus, a transesophageal echocardiogram was recommended if cardioversion was planned within 60 days of randomization. ${ }^{70}$ The ROCKET-AF protocol excluded patients scheduled to have a cardioversion, and few patients underwent the procedure in that trial. ${ }^{14}$ In ARISTOTLE, patients received blinded oral anticoagulation within therapeutic INR ranges for at least 3 weeks prior to elective cardioversion, and transesophageal echocardiography was used to evaluate patients at higher risk of left atrial appendage thrombus. ${ }^{13}$

Trial subanalyses suggest that such approaches were sufficient. A post hoc analysis of patients who had undergone cardioversion in RE-LY found no significant differences between dabigatran and warfarin for the risk of stroke or systemic embolism or the risk of major bleeding within 30 days of the procedure. ${ }^{71}$ A ROCKET-AF subanalysis that adjusted for baseline differences showed that the long-term risks of stroke or systemic embolism, cardiovascular death, and allcause mortality were similar before and after cardioversion or AF ablation in rivaroxaban-treated and warfarin-treated patients. ${ }^{59}$ Finally, an ARISTOTLE subanalysis found very low rates of major clinical events (stroke, systemic embolism, MI, major bleeding) during the first 90 days after cardioversion, and results were similar in apixaban-treated and warfarin-treated patients. ${ }^{72}$

The EHRA guide suggests that, as no anticoagulation assay is available for the NOACs to provide information on anticoagulation over the 3 weeks prior to cardioversion, clinicians should explicitly ask patients about adherence over the weeks prior to procedures and document their answers. ${ }^{22}$ If doubts arise regarding patient compliance, then transesophageal echocardiography should be considered prior to cardioversion.

\section{Percutaneous coronary intervention}

Possibly the most challenging situation for cardiologists is how to use NOACs in clinical conditions where coadministration of antiplatelet therapy is required (ie, dual or triple therapy), especially after percutaneous coronary intervention. The 2014 American College of Cardiology/American Heart Association/Heart Rhythm Society guidelines recommend that bare metal stents may be considered in patients with AF undergoing percutaneous coronary intervention to minimize the required duration of dual antiplatelet therapy, and that anticoagulation may be interrupted at the time of the procedure to reduce the risk of bleeding at the site of peripheral arterial puncture. ${ }^{62}$

In a subanalysis of RE-LY, patients on dual antiplatelet therapy and dabigatran had a $43 \%$ higher risk of major bleeding than patients not taking any antiplatelet therapies. ${ }^{26}$ Since the RE-LY trial results were published, new antiplatelet 
therapies have emerged, including ticagrelor and prasugrel; however, the impact of these agents on increased bleeding risk in patients concurrently taking dabigatran is unknown. In general, for patients requiring dual antiplatelet therapy, exposure to triple therapy should be limited when possible by encouraging the use of bare metal stents in appropriate patients. Notably, the prescribing information for the NOACs warn of an increased risk of bleeding when they are used with antiplatelet therapies. ${ }^{6-8}$ With greater use of NOACs in clinical practice, studies will address these issues and provide guidance about appropriate use of NOACs in select populations. Reducing the time patients are exposed to either dual or triple therapy should be a key consideration for clinicians when selecting from among the different antithrombotic agents. ${ }^{22}$

\section{Patient selection}

It is critical to select appropriate patients for treatment with NOACs to reduce the risk of adverse events. A thorough patient history is imperative, including a review of prior bleeding episodes from any source. Reconciliation of all prescription and nonprescription medications and a current assessment of kidney function are essential to avoiding concomitant drug interactions (Table 1). These steps will help identify which patients are candidates for NOACs and reduce the risk of major adverse events, particularly bleeding complications.

Whether a patient with a stable INR should transition to NOAC treatment is an evolving clinical question, and decisions related to patient selection are probably least relevant for patients who are on steady doses of a VKA without wide fluctuations in their INR. A center-based subanalysis of RE-LY found that when warfarin-treated patients had a time-in-therapeutic range of $>65.5 \%$, dabigatran was no longer superior in terms of risk of stroke or systemic embolism. ${ }^{73}$ Rivaroxaban was not superior to warfarin for the prevention of stroke or systemic embolism; however, the treatment effect of rivaroxaban compared with warfarin was consistent across center-based time-in-therapeutic range quartiles. ${ }^{14}$ An analysis using predicted center-based and individual time-in-therapeutic ranges found that, regardless of the quality of INR control, apixaban offered a benefit versus warfarin with respect to risk of stroke or systemic embolism, bleeding, and mortality. ${ }^{74}$ Some argue that warfarin is associated with a much higher risk of intracranial hemorrhage, which justifies switching such patients to one of the NOACs, and although this is a valid point, the overall risk of intracranial hemorrhage is relatively low (Table 3). ${ }^{11-15}$ Others contend that even well controlled, younger warfarin-treated patients should be switched to a NOAC because of the substantially reduced risk of devastating intracranial hemorrhage.

The 2014 guidelines from the American College of Cardiology/American Heart Association recommend that in patients with AF, antithrombotic therapy should be individualized based on shared decision-making after discussion about the absolute and relative risks of stroke and bleeding and the patient's values and preferences. ${ }^{62}$ A focused 2012 update of the European Society of Cardiology guidelines for management of AF states that the approved NOACs are broadly preferable to VKAs in the majority of patients with nonvalvular AF when used as studied in clinical trials. ${ }^{75}$ The wide therapeutic window and convenience of no routine monitoring make the NOACs desirable first-line options; however, patients taking these drugs still require close follow-up, with frequent renal function assessments to ensure their safe use. The EHRA recommends that patients on NOACs should be assessed every 3 months, with their first follow-up one month after initiation of therapy. ${ }^{22}$

\section{Dabigatran}

Data from RE-LY and subsequent postmarketing analyses have revealed that elderly patients and those with renal dysfunction are most susceptible to bleeding. ${ }^{11,23,24,26}$ Gastrointestinal bleeding appears to be the most common serious side effect of dabigatran therapy. ${ }^{11,26}$ Careful screening of patients for a history of gastrointestinal bleeding and current use of nonsteroidal anti-inflammatory drugs, including aspirin, is essential. Additionally, thorough education about storage and handling of dabigatran is imperative. Patient education on the importance of adherence to dosing schedules is important, as shown by a cross-sectional cohort study of 103 patients who had been treated with dabigatran for at least 3 months, which found that $12 \%$ of patients had inadequate adherence. ${ }^{76}$ Based on current evidence, the best candidates for this treatment include patients $<75$ years of age with normal or near-normal renal function and no history of gastrointestinal bleeding. ${ }^{26}$ A nationwide study of patients with AF in Denmark found that deviations from the recommended use of dabigatran were frequent, and that treatment of VKA-naive patients with dabigatran was safe; however, there was an increased risk of thromboembolism and bleeding in VKA-experienced patients taking dabigatran. ${ }^{77}$ The authors concluded that this may be due to inappropriate patient selection or transitioning patients from VKA therapy to dabigatran. ${ }^{77}$ 


\section{Rivaroxaban}

Rivaroxaban is also an alternative to warfarin in appropriately selected patients with nonvalvular AF. ${ }^{75}$ Two prespecified secondary analyses of ROCKET-AF found older age and decreased renal function to be associated with a higher bleeding risk; however, no differences were seen between rivaroxaban-treated and warfarin-treated patients. ${ }^{32,78}$ In bleeding patients with moderately impaired renal function, critical organ (intracranial, spinal, ocular, pericardial, articular, retroperitoneal, or intramuscular with compartment syndrome ${ }^{79}$ ) and fatal bleeding were less frequent with rivaroxaban than with warfarin. ${ }^{32,78}$ Because gastrointestinal bleeding is more frequent in patients treated with rivaroxaban than in those treated with warfarin, ${ }^{14}$ careful screening of patients for a history of prior bleeding and concurrent nonsteroidal antiinflammatory drug or antiplatelet therapy is vital.

Patients unable to tolerate dabigatran because of side effects, such as dyspepsia, are suitable candidates for rivaroxaban. Furthermore, patients with adherence issues on twice-daily medication schedules may be potential candidates for rivaroxaban. Based on the available clinical data, ${ }^{8}$ patients younger than 75 years of age with good renal function and no history of gastrointestinal bleeding are the most appropriate candidates for rivaroxaban.

\section{Apixaban}

Apixaban may have the best efficacy and safety profile among the NOACs based on currently available data. Apixaban appears to carry a lower risk of major bleeding when compared with warfarin for both older patients and those with renal impairment. ${ }^{13,34}$ Data indicate that patients who are most susceptible to stroke, including older patients (age $>75$ years) and those with moderately impaired renal function (creatinine clearance $30-50 \mathrm{~mL}$ per minute), may be the best candidates for apixaban. ${ }^{13,34}$

\section{Cost-effectiveness}

Cost is an important factor in the use and selection of NOAC therapies. Many patients requiring anticoagulation are older, on fixed incomes, and already taking a number of prescription medications. ${ }^{80}$ Data comparing the cost-effectiveness of the NOACs are limited; however, several studies have shown reduced costs associated with NOACs in comparison with warfarin ${ }^{81-84} \mathrm{~A}$ study from a US payer perspective investigating medical cost reductions associated with use of individual NOACs (dabigatran, rivaroxaban, and apixaban) showed that use of NOACs may be associated with reduced medical costs relative to warfarin, with apixaban having the most substantial cost reduction. ${ }^{85}$ Additionally, an indirect comparison study has evaluated the pharmacoeconomic impact of apixaban versus dabigatran and rivaroxaban over a lifetime, from a UK National Health Services perspective. ${ }^{86}$ The results showed that apixaban may be a cost-effective alternative to dabigatran $150 \mathrm{mg}$ (reduced to $110 \mathrm{mg}$ in patients aged $\geq 80$ years), dabigatran $110 \mathrm{mg}$, and rivaroxaban $20 \mathrm{mg}$ for the long-term reduction of stroke risk in patients with nonvalvular $\mathrm{AF}^{86}$

\section{Discussion}

Until recently, effective reduction of stroke risk in patients with nonvalvular AF has been limited to the VKAs, but several recently approved NOACs now offer alternative treatment options. While the uptake of these agents has been robust, enthusiasm has been tempered by safety concerns prompted by reports of bleeding complications. Recent registry data found that about half of patients with AF received oral anticoagulant therapy, and among those treated, about 13\% received one of the NOACs. ${ }^{87}$ Despite the known risks associated with VKAs, bleeding complications are common, and clinicians are not usually surprised by these outcomes. Although the risks of bleeding are similar or even lower with NOACs, clinicians with limited clinical exposure to them will need to become more comfortable with these agents and their associated risks. Many advantages distinguish NOACs from VKAs, including fewer drug interactions and no need for food restriction or routine anticoagulant monitoring; however, issues similar to those seen with VKAs exist with the NOACs, including how to safely discontinue treatment and when to use bridging therapy or make dose adjustments. Thus, greater oversight of NOAC treatment may be necessary initially until clinicians become familiar with their use. Expanding the role of anticoagulation clinics may help reduce the occurrence of some bleeding events by creating systematic oversight of these agents in clinical practice.

Based on current data for the NOACs and because of the many unresolved clinical issues, clinicians should employ a stepwise approach when using these agents for patients with nonvalvular AF. Appropriate patient selection is critical, and requires diligence by the medical provider in eliciting any history of bleeding episodes and thorough medication reconciliation that may alter the therapeutic effects of these new agents.

As clinicians gain experience using these new anticoagulation options to reduce the risk of stroke in patients with nonvalvular AF, they should remind themselves that they are not trying to "hit the ball out of the park" by first using these therapies for their most challenging patients. Instead, they should start these treatments in patients who have nonvalvular 
AF and several stroke risk factors but for whom treatment is expected to be uncomplicated; for example, patients with normal renal function, average body weight, no history of gastrointestinal bleeding, and no concomitant medications associated with drug interactions. Once clinicians have established a comfort level in using NOACs to treat these patients, they can expand to treat a wider patient population with more complex risks.

\section{Summary}

- The wide therapeutic window and convenience of no routine monitoring make the NOACs desirable first-line options

- Patients taking these drugs require close follow-up with frequent renal function assessments to ensure their safe use

- Among patients with nonvalvular AF treated with warfarin or NOACs, bleeding is more common in patients with low body weight, advanced age, and renal impairment

- Care should be taken to ensure continuous anticoagulation when stopping, interrupting, or switching between anticoagulants to avoid an increased risk of stroke

- Practitioners can identify appropriate patients for treatment with NOACs and avoid adverse events by taking a thorough history and performing a comprehensive review of prior bleeding episodes

- The most appropriate candidates for dabigatran or rivaroxaban include patients younger than 75 years with normal or near-normal renal function and no history of gastrointestinal bleeding

- Rivaroxaban should be considered in patients with adherence issues on twice-daily dosing regimens

- The best candidates for apixaban are older patients (age $>75$ years) and those with moderately impaired renal function (creatinine clearance $30-50 \mathrm{~mL}$ per minute).

\section{Disclosure}

Professional medical writing and editorial assistance was provided by Claire Hall and Nicole Draghi of Caudex Medical, and was funded by Bristol-Myers Squibb Company and Pfizer Inc. The author has been a consultant and/or on the advisory boards of AstraZeneca, Bristol-Myers Squibb Company, and Pfizer Inc.

\section{References}

1. Camm AJ, Kirchhof P, Lip GY, et al. Guidelines for the management of atrial fibrillation: the Task Force for the Management of Atrial Fibrillation of the European Society of Cardiology (ESC). Eur Heart J. 2010;31: 2369-2429.
2. Colilla S, Crow A, Simon T, et al. Projected estimates of prevalence and annual growth rate of atrial fibrillation in the United States from a dynamic age-period progression model. Circ Cardiovasc Qual Outcomes. 2012;5:A2056.

3. Wolf PA, Abbott RD, Kannel WB. Atrial fibrillation as an independent risk factor for stroke: the Framingham Study. Stroke. 1991;22:983-988.

4. [No authors listed]. Risk factors for stroke and efficacy of antithrombotic therapy in atrial fibrillation. Analysis of pooled data from five randomized controlled trials. Arch Intern Med. 1994;154:1449-1457.

5. Bristol-Myers Squibb. Coumadin ${ }^{\circledR}$ (prescribing information). Available from: http://packageinserts.bms.com/pi/pi_coumadin.pdf. Accessed October 11, 2013.

6. Boehringer Ingelheim. Pradaxa ${ }^{\circledR}$ (dabigatran etexilate) capsules (prescribing information). Available from: http://bidocs. boehringer-ingelheim.com/BIWebAccess/ViewServlet. ser?docBase=renetnt $\&$ folderPath=/Prescribing\%20Information/PIs/ Pradaxa/Pradaxa.pdf. Accessed September 18, 2013.

7. Bristol-Myers Squibb. Eliquis ${ }^{\circledR}$ (apixaban tablets, prescribing information). Available from: http://packageinserts.bms.com/pi/ pi_eliquis.pdf. Accessed March 20, 2014.

8. Janssen Pharmaceuticals. Xarelto ${ }^{\mathbb{B}}$ (rivaroxaban tablets, prescribing information). Available from: http://www.xareltohcp.com/sites/default/ files/pdf/xarelto_0.pdf\#zoom=100. Accessed October 8, 2013.

9. Mendell J, Zahir H, Matsushima N, et al. Drug-drug interaction studies of cardiovascular drugs involving P-glycoprotein, an efflux transporter, on the pharmacokinetics of edoxaban, an oral factor Xa inhibitor. Am J Cardiovasc Drugs. 2013;13:331-342.

10. Ogata K, Mendell-Harary J, Tachibana M, et al. Clinical safety, tolerability, pharmacokinetics, and pharmacodynamics of the novel factor Xa inhibitor edoxaban in healthy volunteers. J Clin Pharmacol. 2010;50:743-753

11. Connolly SJ, Ezekowitz MD, Yusuf S, et al. Dabigatran versus warfarin in patients with atrial fibrillation. N Engl J Med. 2009;361:1139-1151.

12. Connolly SJ, Eikelboom J, Joyner C, et al. Apixaban in patients with atrial fibrillation. $N$ Engl J Med. 2011;364:806-817.

13. Granger CB, Alexander JH, McMurray JJ, et al. Apixaban versus warfarin in patients with atrial fibrillation. $N$ Engl J Med. 2011;365: 981-992.

14. Patel MR, Mahaffey KW, Garg J, et al. Rivaroxaban versus warfarin in nonvalvular atrial fibrillation. $N$ Engl J Med. 2011;365:883-891.

15. Connolly SJ, Ezekowitz MD, Yusuf S, et al. Newly identified events in the RE-LY trial. N Engl J Med. 2010;363:1875-1876.

16. Giugliano RP, Ruff CT, Braunwald E, et al. Edoxaban versus warfarin in patients with atrial fibrillation. $N$ Engl J Med. 2013;369:2093-2104.

17. Alexander JH, Levy E, Lawrence J, et al. Documentation of study medication dispensing in a prospective large randomized clinical trial: experiences from the ARISTOTLE trial. Am Heart J. 2013;166: 559-565.

18. Food and Drug Administration. Center for Drug Evaluation and Research. Application number 2021550rig1s000. Summary review. Available from: http://www.accessdata.fda.gov/drugsatfda_docs/ nda/2012/202155Orig1s000SumR.pdf. Accessed June 3, 2013.

19. Bristol-Myers Squibb, Pfizer EEIG. Eliquis ${ }^{\circledR}$ (apixaban tablets) Summary of product characteristics. Available from: http://www.ema. europa.eu/docs/en_GB/document_library/EPAR_-_Product_Information/ human/002148/WC500107728.pdf. Accessed June 2, 2014.

20. Uchino K, Hernandez AV. Dabigatran association with higher risk of acute coronary events: meta-analysis of noninferiority randomized controlled trials. Arch Intern Med. 2012;172:397-402.

21. Hohnloser SH, Oldgren J, Yang S, et al. Myocardial ischemic events in patients with atrial fibrillation treated with dabigatran or warfarin in the RE-LY trial. Circulation. 2012;125:669-676.

22. Heidbuchel H, Verhamme P, Alings M, et al. European Heart Rhythm Association Practical Guide on the use of new oral anticoagulants in patients with non-valvular atrial fibrillation. Europace. 2013;15: 625-651. 
23. Harper P, Young L, Merriman E. Bleeding risk with dabigatran in the frail elderly. $N$ Engl J Med. 2012;366:864-866.

24. Legrand M, Mateo J, Aribaud A, et al. The use of dabigatran in elderly patients. Arch Intern Med. 2011;171:1285-1286.

25. Wood S. Deaths prompt dabigatran safety advisory in Japan. Available from: http://www.medscape.com/viewarticle/748143. Accessed April 24, 2014.

26. Eikelboom JW, Wallentin L, Connolly SJ, et al. Risk of bleeding with 2 doses of dabigatran compared with warfarin in older and younger patients with atrial fibrillation: an analysis of the randomized evaluation of long-term anticoagulant therapy (RE-LY) trial. Circulation. 2011;123: $2363-2372$.

27. European Medicines Agency. Questions and answers on the review of bleeding risk with Pradaxa (dabigatran etexilate). Available from: http://www.ema.europa.eu/docs/en_GB/document_library/Medicine_ QA/2012/05/WC500127768.pdf. Accessed November 1, 2012.

28. Southworth MR, Reichman ME, Unger EF. Dabigatran and postmarketing reports of bleeding. N Engl J Med. 2013;368:1272-1274.

29. US Food and Drug Administration. Drug Safety Communication: FDA study of Medicare patients finds risks lower for stroke and death but higher for gastrointestinal bleeding with Pradaxa (dabigatran) compared to warfarin. May 13, 2014. Available from: http://www.fda.gov/downloads/ Drugs/DrugSafety/UCM397606.pdf. Accessed June 4, 2014.

30. Larsen TB, Rasmussen LH, Skjoth F, et al. Efficacy and safety of dabigatran etexilate and warfarin in "real-world" patients with atrial fibrillation: a prospective nationwide cohort study. $J$ Am Coll Cardiol. 2013;61:2264-2273.

31. Ho JC, Chang AM, Yan BP, et al. Dabigatran compared with warfarin for stroke prevention with atrial fibrillation: experience in Hong Kong. Clin Cardiol. 2012;35:E40-E45.

32. Goodman SG, Wojdyla DM, White HD, et al. Predictors of major bleeding risk: insights from the rivaroxaban once-daily oral direct factor Xa inhibition compared with vitamin $\mathrm{K}$ antagonism for prevention of stroke and embolism trial in atrial fibrillation (ROCKET AF). Circulation. 2011;124:A16903.

33. Piccini JP, Garg J, Patel MR, et al. Management of major bleeding events in patients treated with rivaroxaban vs warfarin: results from the ROCKET AF trial. Eur Heart J. 2014;35:1873-1880.

34. Hohnloser SH, Hijazi Z, Thomas L, et al. Efficacy of apixaban when compared with warfarin in relation to renal function in patients with atrial fibrillation: insights from the ARISTOTLE trial. Eur Heart $J$. 2012;33:2821-2830.

35. Hylek EM, Held C, Alexander JH, et al. Major bleeding in patients with atrial fibrillation receiving apixaban or warfarin in the ARISTOTLE trial (Apixaban for Reduction in Stroke and Other Thromboembolic Events in Atrial Fibrillation): predictors, characteristics, and clinical outcomes. J Am Coll Cardiol. 2014;63:2141-2147.

36. Barrett YC, Wang Z, Frost C, Shenker A. Clinical laboratory measurement of direct factor $\mathrm{Xa}$ inhibitors: anti-Xa assay is preferable to prothrombin time assay. Thromb Haemost. 2010;104:1263-1271.

37. Garcia D, Barrett YC, Ramacciotti E, Weitz JI. Laboratory assessment of the anticoagulant effects of the next generation of oral anticoagulants. J Thromb Haemost. 2013;11:245-252.

38. Liesenfeld KH, Schafer HG, Troconiz IF, et al. Effects of the direct thrombin inhibitor dabigatran on ex vivo coagulation time in orthopaedic surgery patients: a population model analysis. Br J Clin Pharmacol. 2006;62:527-537.

39. Samama MM, Martinoli JL, Le Flem L, et al. Assessment of laboratory assays to measure rivaroxaban - an oral, direct factor Xa inhibitor. Thromb Haemost. 2010;103:815-825.

40. Stangier J, Feuring M. Using the HEMOCLOT direct thrombin inhibitor assay to determine plasma concentrations of dabigatran. Blood Coagul Fibrinolysis. 2012;23:138-143.

41. van Ryn J, Ruehl D, Priepke H, et al. Reversibility of the anticoagulant effect of high doses of the direct thrombin inhibitor dabigatran, by recombinant factor VIIa or activated prothrombin complex concentrate. Haematologica. 2008;93:148.
42. Wong PC, Crain EJ, Xin B, et al. Apixaban, an oral, direct and highly selective factor Xa inhibitor: in vitro, antithrombotic and antihemostatic studies. J Thromb Haemost. 2008;6:820-829.

43. Hawes EM, Deal AM, Funk-Adcock D, et al. Performance of coagulation tests in patients on therapeutic doses of dabigatran: a cross-sectional pharmacodynamic study based on peak and trough plasma levels. J Thromb Haemost. 2013;11:1493-1502.

44. Becker RC, Yang H, Barrett Y, et al. Chromogenic laboratory assays to measure the factor Xa-inhibiting properties of apixaban - an oral, direct and selective factor Xa inhibitor. J Thromb Thrombolysis. 2011;32:183-187.

45. Samama MM, Amiral J, Guinet C, et al. An optimised, rapid chromogenic assay, specific for measuring direct factor Xa inhibitors (rivaroxaban) in plasma. Thromb Haemost. 2010;104:1078-1079.

46. Miyares MA, Davis K. Newer oral anticoagulants: a review of laboratory monitoring options and reversal agents in the hemorrhagic patient. Am J Health Syst Pharm. 2012;69:1473-1484.

47. Wolzt M, Weltermann A, Nieszpaur-Los M, et al. Studies on the neutralizing effects of protamine on unfractionated and low molecular weight heparin (Fragmin) at the site of activation of the coagulation system in man. Thromb Haemost. 1995;73:439-443.

48. Zhou W, Schwarting S, Illanes S, et al. Hemostatic therapy in experimental intracerebral hemorrhage associated with the direct thrombin inhibitor dabigatran. Stroke. 2011;42:3594-3599.

49. Food and Drug Administration. Advisory Committee Briefing Book. Rivaroxaban for the prophylaxis of deep vein thrombosis (DVT) and pulmonary embolism (PE) in patients undergoing hip or knee replacement surgery. Available from: http://www.fda.gov/downloads/AdvisoryCommittees/ CommitteesMeetingMaterials/Drugs/CardiovascularandRenalDrugsAd visoryCommittee/UCM138385.pdf. Accessed February 19, 2012.

50. Wang X, Mondal S, Wang J, et al. Effect of activated charcoal on apixaban pharmacokinetics in healthy subjects. Am J Cardiovasc Drugs. 2014;14:147-154.

51. Eerenberg ES, Kamphuisen PW, Sijpkens MK, et al. Reversal of rivaroxaban and dabigatran by prothrombin complex concentrate: a randomized, placebo-controlled, crossover study in healthy subjects. Circulation. 2011;124:1573-1579.

52. Marlu R, Hodaj E, Paris A, et al. Effect of non-specific reversal agents on anticoagulant activity of dabigatran and rivaroxaban: a randomised crossover ex vivo study in healthy volunteers. Thromb Haemost. 2012;108:217-224.

53. Escolar G, Arellano-Rodrigo E, Reverter JC, et al. Reversal of apixaban induced alterations of hemostasis by different coagulation factor concentrates: studies in vitro with circulating human blood. Circulation. 2012;126:520-521.

54. Crowther M, Kitt M, Lorenz T, et al. A phase 2 randomized, double-blind, placebo-controlled trial of PRT064445, a novel, universal antidote for direct and indirect factor Xa inhibitors. J Thromb Haemost. 2013;11 Suppl 2:30.

55. Crowther M, Mathur V, Kitt M, et al. A phase 2 randomized, double-blind, placebo-controlled trial demonstrating reversal of rivaroxaban-induced anticoagulation in healthy subjects by andexanet alfa (PRT064445), an antidote for FXa inhibitors. Abstract presented at the 55th Annual Meeting of the American Society of Hematology, December 7-10, 2013, New Orleans, LA, USA.

56. Granger C, Alexander JH, Hanna M, et al. Events after discontinuation of randomized treatment at the end of the ARISTOTLE trial. Eur Heart J. 2012;33 Suppl:685-686.

57. Lakkireddy D, Reddy YM, Di Biase L, et al. Feasibility and safety of dabigatran versus warfarin for periprocedural anticoagulation in patients undergoing radiofrequency ablation for atrial fibrillation: results from a multicenter prospective registry. J Am Coll Cardiol. 2012;59:1168-1674.

58. Patel MR, Hellkamp AS, Lokhnygina Y, et al. Outcomes of discontinuing rivaroxaban compared with warfarin in patients with nonvalvular atrial fibrillation: analysis from the ROCKET AF trial (rivaroxaban once-daily, oral, direct factor Xa inhibition compared with vitamin $\mathrm{K}$ antagonism for prevention of stroke and embolism trial in atrial fibrillation). J Am Coll Cardiol. 2013;61:651-658. 
59. Piccini JP, Stevens SR, Lokhnygina Y, et al. Outcomes after cardioversion and atrial fibrillation ablation in patients treated with rivaroxaban and warfarin in the ROCKET AF trial. J Am Coll Cardiol. 2013;61: 1998-2006.

60. Douketis JD, Spyropoulos AC, Spencer FA, et al. Perioperative Management of Antithrombotic Therapy: Antithrombotic Therapy and Prevention of Thrombosis, 9th ed: American College of Chest Physicians EvidenceBased Clinical Practice Guidelines. Chest. 2012;141:e326S-e350S.

61. Larsen TB, Rasmussen LH, Gorst-Rasmussen A, et al. Myocardial ischemic events in "real world" patients with atrial fibrillation treated with dabigatran or warfarin. Am J Med. 2014;127:329-336. e4.

62. January CT, Wann LS, Alpert JS, et al. 2014 AHA/ACC/HRS Guideline for the Management of Patients With Atrial Fibrillation: Executive Summary: A Report of the American College of Cardiology/ American Heart Association Task Force on Practice Guidelines and the Heart Rhythm Society. J Am Coll Cardiol. 2014;pii: S0735-1097(14)01740-01749.

63. Eitel C, Koch J, Sommer P, et al. Novel oral anticoagulants in a real-world cohort of patients undergoing catheter ablation of atrial fibrillation. Europace. 2013;15:1587-1593.

64. Providencia R, Marijon E, Albenque JP, et al. Rivaroxaban and dabigatran in patients undergoing catheter ablation of atrial fibrillation. Europace. 2014;16:1137-1144.

65. Sardar P, Nairooz R, Chatterjee S, et al. Meta-analysis of risk of stroke or transient ischemic attack with dabigatran for atrial fibrillation ablation. Am J Cardiol. 2014;113:1173-1177.

66. Stepanyan G, Badhwar N, Lee RJ, et al. Safety of new oral anticoagulants for patients undergoing atrial fibrillation ablation. J Interv Card Electrophysiol. 2014;4:33-38.

67. Winkle RA, Mead RH, Engel G, et al. The use of dabigatran immediately after atrial fibrillation ablation. J Cardiovasc Electrophysiol. 2012;23:264-268.

68. Healey JS, Eikelboom J, Douketis J, et al. Periprocedural bleeding and thromboembolic events with dabigatran compared with warfarin: results from the Randomized Evaluation of Long-Term Anticoagulation Therapy (RE-LY) randomized trial. Circulation. 2012;126: 343-348.

69. Lopes RD, Garcia DA, Wojdyla DM. Use of apixaban and warfarin in patients undergoing invasive procedures: insights from ARISTOTLE. Eur Heart J. 2013;34 Suppl:98-99.

70. Ezekowitz MD, Connolly S, Parekh A, et al. Rationale and design of RE-LY: randomized evaluation of long-term anticoagulant therapy, warfarin, compared with dabigatran. Am Heart J. 2009;157: 805-810.

71. Nagarakanti R, Ezekowitz MD, Oldgren J, et al. Dabigatran versus warfarin in patients with atrial fibrillation: an analysis of patients undergoing cardioversion. Circulation. 2011;123:131-136.

72. Flaker G, Lopes RD, Al-Khatib SM, et al. Apixaban and warfarin are associated with a low risk of stroke following cardioversion for atrial fibrillation: results from the ARISTOTLE trial. Eur Heart $J$. 2012;33:686.

73. Wallentin L, Yusuf S, Ezekowitz MD, et al. Efficacy and safety of dabigatran compared with warfarin at different levels of international normalised ratio control for stroke prevention in atrial fibrillation: an analysis of the RE-LY trial. Lancet. 2010;376:975-983.
74. Wallentin L, Lopes RD, Hanna M, et al. Efficacy and safety of apixaban compared with warfarin at different levels of predicted international normalized ratio control for stroke prevention in atrial fibrillation. Circulation. 2013;127:2166-2176.

75. Camm AJ, Lip GY, De Caterina R, et al. 2012 focused update of the ESC guidelines for the management of atrial fibrillation: an update of the 2010 ESC guidelines for the management of atrial fibrillation. Developed with the special contribution of the European Heart Rhythm Association. Eur Heart J. 2012;33:2719-2747.

76. Schulman S, Shortt B, Robinson M, Eikelboom JW. Adherence to anticoagulant treatment with dabigatran in a real-world setting. J Thromb Haemost. 2013;11:1295-1299.

77. Sorensen R, Gislason G, Torp-Pedersen C, et al. Dabigatran use in Danish atrial fibrillation patients in 2011: a nationwide study. BMJ Open. 2013;3.

78. Fox KA, Piccini JP, Wojdyla D, et al. Prevention of stroke and systemic embolism with rivaroxaban compared with warfarin in patients with non-valvular atrial fibrillation and moderate renal impairment. Eur Heart J. 2011;32:2387-2394.

79. Schulman S, Kearon C, the Subcommittee on Control of Anticoagulation of the Scientific and Standardization Committee of the International Society on Thrombosis and Haemostasis. Definition of major bleeding in clinical investigations of antihemostatic medicinal products in non-surgical patients. $J$ Thromb Haemost. 2005;3: 692-694.

80. Gu Q, Dillion CF, Burt VL. NCHC Data brief: prescription drug use continues to increase: US prescription drug data for 2007-2008. Available from: http://www.cdc.gov/nchs/data/databriefs/db42.htm. Accessed June 6, 2013

81. Freeman JV, Zhu RP, Owens DK, et al. Cost-effectiveness of dabigatran compared with warfarin for stroke prevention in atrial fibrillation. Ann Intern Med. 2011;154:1-11.

82. Kamel H, Easton JD, Johnston SC, Kim AS. Cost-effectiveness of apixaban vs warfarin for secondary stroke prevention in atrial fibrillation. Neurology. 2012;79:1428-1434.

83. Lee S, Anglade MW, Meng J, et al. Cost-effectiveness of apixaban compared with aspirin for stroke prevention in atrial fibrillation among patients unsuitable for warfarin. Circ Cardiovasc Qual Outcomes. 2012;5:472-479.

84. Lee S, Anglade MW, Pham D, et al. Cost-effectiveness of rivaroxaban compared to warfarin for stroke prevention in atrial fibrillation. Am J Cardiol. 2012;110:845-851.

85. Deitelzweig S, Amin A, Jing Y, et al. Medical cost reductions associated with the usage of novel oral anticoagulants vs warfarin among atrial fibrillation patients, based on the RE-LY, ROCKET-AF and ARISTOTLE trials. $J$ Med Econ. 2012;15:776-785.

86. Lip GYH, Kongnakorn T, Phatak H, et al. Cost-effectiveness of apixaban versus other new oral anticoagulants for stroke prevention in atrial fibrillation. Clin Ther. 2014;36:192-210.

87. American College of Cardiology. Registry data shows early patterns for new atrial fibrillation treatments. Available from: http://www.cardiosource.org/News-Media/Media-Center/News-Releases/2012/08/ PINN-AF.aspx. Accessed October 15, 2012.
Vascular Health and Risk Management

\section{Publish your work in this journal}

Vascular Health and Risk Management is an international, peerreviewed journal of therapeutics and risk management, focusing on concise rapid reporting of clinical studies on the processes involved in the maintenance of vascular health; the monitoring, prevention and treatment of vascular disease and its sequelae; and the involvement of

\section{Dovepress}

metabolic disorders, particularly diabetes. This journal is indexed on PubMed Central and MedLine. The manuscript management system is completely online and includes a very quick and fair peer-review system, which is all easy to use. Visit http://www.dovepress.com/ testimonials.php to read real quotes from published authors. 